\title{
Mean-field yrast spectrum of a two-component Bose gas in ring geometry: persistent currents at higher angular momentum
}

\author{
Z. Wu and E. Zaremba \\ Department of Physics, Queen's University, Kingston, ON,Canada
}

(Dated: November 2, 2018)

\begin{abstract}
We use analytic soliton solutions of a two-component Bose gas in the ring geometry to analyze the mean-field yrast spectrum of the system. We find that the spectrum exhibits a surprisingly rich structure as a result of an intricate interplay of interparticle interactions and population imbalance. We discuss the implication of these results in regard to the possibility of persistent currents at higher angular momenta.

PACS numbers: 67.85.De, 03.75.Kk, 03.75.Mn, 05.30.Jp
\end{abstract}

\section{INTRODUCTION}

According to Bloch [1], the possibility of persistent currents in a bosonic system in the ring geometry is determined by the yrast spectrum [2]. which is defined by the lowest energy state for a given total angular momentum $L$. For a single-species system, Bloch [1] showed that the yrast spectrum takes the form

$$
E_{0}(L)=\frac{L^{2}}{2 M_{T} R^{2}}+e_{0}(L)
$$

where $M_{T}=N M$ is the total mass of the system, $R$ is the radius of the ring on which the bosons reside, and $e_{0}(L)$ is a periodic function of the angular momentum with the properties $e_{0}(-L)=e_{0}(L)$ and $e_{0}(L+\nu N \hbar)=e_{0}(L)$. Here, $N$ is the number of particles of mass $M$ and $\nu$ is an integer. Bloch argued that the system can support persistent currents at some non-zero angular momentum $L_{0}$ if $E_{0}\left(L_{0}\right)$ is a local minimum.

Bloch's analysis can be extended to a binary mixture containing $N_{A}$ particles of mass $M_{A}$ and $N_{B}$ particles of mass $M_{B}$. In general, the function $e_{0}(L)$, where $L$ is again the total angular momentum, exhibits a periodicity only if the mass ratio $M_{A} / M_{B}$ is a rational number. For the special case $M_{A}=M_{B}$, which is realizable with bosons in different hyperfine states, $e_{0}(L)$ retains the same periodicity as for the single-species system, but with $N=N_{A}+N_{B}$. In terms of $l=L / N \hbar$, the angular momentum per particle in units of $\hbar$, one has $e_{0}(l+\nu)=e_{0}(l)$ and $e_{0}(-l)=e_{0}(l)$. In this case, the behaviour of $e_{0}(l)$ in the interval $0 \leq l \leq 1 / 2$ is sufficient to decide on the possibility of persistent currents.

For a single-spieces system of bosons interacting via a contact interaction, the yrast spectrum was deterimined by Lieb [3]. The spectrum is defined by the socalled type-II excitations which have been identified as soliton-like by comparing their energy with those of soliton states as obtained from a mean-field analysis [4 6 ]. Although a similar identification has not been established for the binary system, it has generally been assumed that a mean-field analysis would provide a reasonably accurate approximation of the yrast spectrum in this case as well.
Determination of such a mean-field spectrum requires the knowledge of soliton solutions to coupled GrossPitaevskii equations. General solutions of this kind, expressed in terms of Jacobi elliptic functions, were first obtained by Porubov and Parker [7] in the context of solving a Manokov system of coupled nonlinear Schrödinger equations. Smyrnakis et al. [8, 9] found these same solutions in the context of the binary bosonic mixture in the ring geometry and applied them to determine the meanfield yrast spectrum of a system with relatively weak interaction strength and small population imbalance. One of the purposes of this paper is to present a complete analysis of the mean-field yrast spectrum of a binary system with arbitrary interaction strength and population imbalance. We find that the yrast spectrum reveals a surprisingly rich structure, not seen in Refs. 8, 9], as a result of the interplay of interaction and population imbalance.

More importantly, our findings of the yrast spectrum provide a definitive answer to the question as to whether persistent currents occur at higher angular momenta. The first study of this kind for a binary system was carried out in Ref. 10] wherein the authors determined a limited portion of the yrast spectrum. With this information, the authors concluded that an arbitrarily small concentration of the minority component, $x_{B}$, would disrupt the possibility of persistent currents at higher angular momenta. On the other hand, we argued in a recent paper [11] that the yrast spectrum must revert, on the basis of continuity, to that of the single-species system in the $x_{B} \rightarrow 0$ limit. A semi-quantitative calculation of the yrast spectrum supported this physically obvious expectation, but a definitive explanation of how this occurred was not provided.

The second purpose of the present paper is to analyze the $x_{B} \rightarrow 0$ limit in more detail using the analytic soliton solutions. This analysis confirms that the single-species results are recovered in the $x_{B} \rightarrow 0$ limit. However, this limit is reached in a surprisingly complex way with the emergence of distinct soliton-like states in different ranges of the angular momentum. Furthermore, we obtain a simple, analytic formula for the critical interaction strength above which persistent currents are shown to ex- 
ist at arbitrarily high angular momenta. This provides an explicit affirmation of the general argument we gave in Ref. [11].

The paper is organized as follows. In Sec. II we review the soliton solutions of the GP equations for a binary mixture. In Sec. III we present a detailed analysis of the mean-field yrast spectrum for arbitrary interaction strengths and minority concentrations $x_{B}$. This information is then used to investigate the possibility of persistent currents at higher angular momenta and explain in detail how the single-species limit is recovered for $x_{B} \rightarrow 0$. Our conclusions are presented in the final section.

\section{TWO-COMPONENT MODEL AND THE ANSATZ SOLITON-TRAIN SOLUTIONS}

We consider an equal-mass, two-component Bose gas in the one-dimensional ring geometry with repulsive interactions described by the following Hamiltonian

$$
\hat{H}=-\sum_{i s} \frac{\hbar^{2}}{2 M R^{2}} \frac{\partial^{2}}{\partial \theta_{s i}^{2}}+\sum_{s s^{\prime}} \sum_{i j} \frac{U}{4 \pi R} \delta\left(\theta_{s i}-\theta_{s^{\prime} j}\right),
$$

where $U$ is the effective interaction strength and $s=A, B$ distinguishes the two atomic species. The numbers of atoms in the two components, $N_{A}$ and $N_{B}$ respectively, are fixed and for definiteness we take $N_{A} \geq N_{B}$.

Within mean-field theory, the system is described in terms of condensate wave functions $\psi_{A}(\theta)$ and $\psi_{B}(\theta)$ which are taken to have unit normalization $\int_{0}^{2 \pi} d \theta\left|\psi_{s}(\theta)\right|^{2}=1$. The mean-field energy functional, in units of $N \hbar^{2} /\left(2 M R^{2}\right)$ where $N=N_{A}+N_{B}$, is given by

$$
\bar{E}\left[\psi_{A}, \psi_{B}\right]=\sum_{s} x_{s} \int_{0}^{2 \pi} d \theta\left|\frac{d \psi_{s}}{d \theta}\right|^{2}+\pi \gamma \int_{0}^{2 \pi} d \theta \rho^{2}(\theta),
$$

where $x_{s}=N_{s} / N, \gamma=N M R^{2} U / \pi \hbar^{2}$ and $\rho(\theta)=$ $x_{A}\left|\psi_{A}\right|^{2}+x_{B}\left|\psi_{B}\right|^{2}$ is the normalized number density. Our objective is to minimize the energy with respect to $\psi_{s}(\theta)$ subject to the two normalization contraints and the angular momentum constraint

$$
\bar{L} \equiv \frac{1}{i} \sum_{s} x_{s} \int_{0}^{2 \pi} d \theta \psi_{s}^{*} \frac{d}{d \theta} \psi_{s}=l .
$$

This can be achieved by minimizing the functional

$$
\bar{F}\left[\psi_{A}, \psi_{B}\right]=\bar{E}-\Omega \bar{L}-\sum_{s} x_{s} \mu_{s} \int_{0}^{2 \pi} d \theta\left|\psi_{s}(\theta)\right|^{2},
$$

where $\Omega$ and $\mu_{s}$ are (dimensionless) Lagrange multipliers. The variation of $\bar{F}$ with respect to $\psi_{s}$ leads to the coupled time-independent Gross-Pitaevskii (GP) equations

$$
-\psi_{s}^{\prime \prime}(\theta)+i \Omega \psi_{s}^{\prime}(\theta)+2 \pi \gamma \rho(\theta) \psi_{s}(\theta)=\mu_{s} \psi_{s}(\theta) .
$$

These same equations can be obtained from the timedependent GP equations by assuming solutions of the form $\psi_{s}(\theta, t)=\psi_{s}(\theta-\Omega t) e^{-i \mu_{s} t}$, where $\Omega$ is the angular velocity of the soliton, in units of $N \hbar /\left(2 M R^{2}\right)$. Although Eq. (6) can be viewed as representing the system in a rotating frame of reference in which the soliton is at rest, we emphasize that $\Omega$ is not a free parameter but is determined by the soliton state itself.

The lowest energy soliton solution consistent with the angular momentum constraint in Eq. (4) defines the mean-field yrast spectrum [2], $\bar{E}_{0}(l)$, as a function of the angular momentum per particle, $l$. The fact that the soliton is a variational minimum of Eq. (5D) leads to the relation

$$
\Omega=\frac{\partial \bar{E}_{0}(l)}{\partial l} .
$$

Eq. (7) is an important and useful relation which shows that the slope of the yrast spectrum $\bar{E}_{0}(l)$ is simply the angular velocity $\Omega$.

To solve the coupled equations in Eq. (6) we make use of the modulus-phase representation

$$
\psi_{s}(\theta)=\sqrt{\rho_{s}(\theta)} e^{i \varphi_{s}(\theta)} .
$$

The single-valuedness of the wave function $\psi_{s}(\theta)$ implies the following boundary conditions

$$
\begin{aligned}
\rho_{s}(\theta+2 \pi)-\rho_{s}(\theta) & =0 \\
\varphi_{s}(\theta+2 \pi)-\varphi_{s}(\theta) & =2 \pi J_{s}, \quad J_{s}=0, \pm 1, \pm 2, \cdots
\end{aligned}
$$

where the integers $J_{s}$ are referred to as phase winding numbers.

Substituting Eq. (8) into Eq. (6), one obtains

$$
-\left(\sqrt{\rho_{s}}\right)^{\prime \prime}+\left(\varphi_{s}^{\prime}-\Omega\right) \varphi_{s}^{\prime} \sqrt{\rho_{s}}+2 \pi \gamma \rho \sqrt{\rho_{s}}=\mu_{s} \sqrt{\rho_{s}}
$$

and

$$
\sqrt{\rho_{s}} \varphi_{s}^{\prime \prime}+\left(2 \varphi_{s}^{\prime}-\Omega\right)\left(\sqrt{\rho_{s}}\right)^{\prime}=0 .
$$

The solution to Eq. (12) is given by

$$
\varphi_{s}^{\prime}(\theta)=\frac{W_{s}}{2 \rho_{s}}+\frac{\Omega}{2},
$$

where $W_{s}$ is an integration constant to be determined. Physically, $\varphi_{s}^{\prime}(\theta)$ gives the superfluid velocity field of each component. Using Eq. (4) and Eq. (13), one finds that the angular momentum per particle can be written as

$$
l=x_{A} l_{A}+x_{B} l_{B},
$$

where

$$
l_{s}=\pi W_{s}+\frac{\Omega}{2}
$$

is the angular momentum per particle of each species. Finally, inserting Eq. (13) into Eq. (11), one obtains

$$
\frac{1}{2} \rho_{s} \rho_{s}^{\prime \prime}-\frac{1}{4}\left(\rho_{s}^{\prime}\right)^{2}-2 \pi \gamma \rho \rho_{s}^{2}+\tilde{\mu}_{s} \rho_{s}^{2}-\frac{W_{s}^{2}}{4}=0,
$$


where $\tilde{\mu}_{s}=\mu_{s}+\Omega^{2} / 4$. These are the coupled equations that determine the densities of the two components.

The coupled equations in Eq. (16) admit solutions of the form [7 9

$$
\rho_{B}=c_{B}\left(1+c_{A}^{-1} \rho_{A}\right),
$$

where $c_{s}$ are coefficients to be determined. Since $\rho_{A}$ and $\rho_{B}$ are normalized to unity, an angular integration of Eq. (17) yields the following relationship between $c_{A}$ and $c_{B}$,

$$
2 \pi c_{B}+c_{B} c_{A}^{-1}=1 .
$$

Using the ansatz in Eq. (17) to eliminate $\rho_{B}$ for $s=A$ and $\rho_{A}$ for $s=B$, respectively, from Eq. (16), one finds

$$
\frac{1}{2} \rho_{s} \rho_{s}^{\prime \prime}-\frac{1}{4}\left(\rho_{s}^{\prime}\right)^{2}-2 \pi \gamma_{s} \rho_{s}^{3}+\bar{\mu}_{s} \rho_{s}^{2}-\frac{W_{s}^{2}}{4}=0 .
$$

Here

$$
\gamma_{s}=c_{s}^{-1}\left(c_{A} x_{A}+c_{B} x_{B}\right) \gamma
$$

and

$$
\bar{\mu}_{s}=\tilde{\mu}_{s} \mp 2 \pi \gamma c_{\bar{s}} x_{\bar{s}},
$$

where $\bar{s}$ denotes the complementary species to $s$ and the - (+) sign corresponds to the $A(B)$ component. It is clear from Eq. (20) that the effective interactions $\gamma_{s}$ depend on the coefficients $c_{S}$ only only through the ratio $r \equiv c_{B} / c_{A}$ and that $\gamma_{A} / \gamma_{B}=r$. Having introduced this ratio, we observe that the normalization relation in Eq. (18) implies that

$$
c_{A}=\frac{1-r}{2 \pi r}, \quad c_{B}=\frac{1-r}{2 \pi},
$$

and that the density ansatz in Eq. (17) takes the form

$$
\rho_{B}=\frac{1-r}{2 \pi}+r \rho_{A}
$$

The ratio $r$ plays a central role in the subsequent analysis.

Equation (19) indicates that the ansatz in Eq. (17) reduces the coupled system to two independent equations, one for each species with a modified effective interaction. Although independent, the solutions must nevertheless be consistent with the starting ansatz. As we shall see, there is indeed sufficient freedom in the form of the two independent solutions to ensure that Eq. (17) is satisfied. We refer to this condition as the self-consistency requirement. (For an alternative method of checking selfconsistency, see Refs. 7-9].) We finally note that the ansatz allows one to express the energy per particle as

$$
\bar{E}=x_{A} \bar{E}_{A}+x_{B} \bar{E}_{B}-2 \pi^{2} \gamma x_{A} x_{B} c_{A} c_{B},
$$

where

$$
\bar{E}_{s}=\int_{0}^{2 \pi} d \theta\left|\frac{d \psi_{s}}{d \theta}\right|^{2}+\pi \gamma_{s} \int_{0}^{2 \pi} d \theta \rho_{s}^{2}(\theta) .
$$

Despite its appearance, Eq. (24) accounts fully for the interactions between the two atomic species.

There are two classes of solutions to Eq. (19), one corresponding to $c_{A} x_{A}+c_{B} x_{B}=0$ and the other to $c_{A} x_{A}+c_{B} x_{B} \neq 0$. We analyze these two classes in turn.

\section{Class (i) solutions: $c_{A} x_{A}+c_{B} x_{B}=0$}

In this case, $\gamma_{A}=\gamma_{B}=0$ and the system is effectively reduced to two non-interacting species. Since $r=-x_{A} / x_{B}, \mathrm{Eq}$. (22) gives

$$
c_{A}=-\frac{1}{2 \pi x_{A}}, \quad c_{B}=\frac{1}{2 \pi x_{B}}
$$

and Eq. (23) implies that the total density is a constant, $\rho(\theta)=1 / 2 \pi$. For $\gamma_{s}=0$, Eq. (19) becomes

$$
\frac{1}{2} \rho_{s} \rho_{s}^{\prime \prime}-\frac{1}{4}\left(\rho_{s}^{\prime}\right)^{2}+\bar{\mu}_{s} \rho_{s}^{2}-\frac{W_{s}^{2}}{4}=0 .
$$

Defining the function $y_{s}=-\rho_{s}^{\prime \prime} / 4+\bar{\mu}_{s} \rho_{s}^{2}-W_{s}^{2} / 4$, Eq. (27) is equivalent to

$$
\frac{y_{s}^{\prime}}{y_{s}}=\frac{\rho_{s}^{\prime}}{\rho_{s}}
$$

which, via an integration, leads to

$$
\frac{1}{4}\left(\rho_{s}^{\prime}\right)^{2}+\bar{\mu}_{s} \rho_{s}^{2}+V_{s} \rho_{s}+\frac{W_{s}^{2}}{4}=0,
$$

where $V_{s}$ is an integration constant.

Using the boundary condition in Eq. (9) and the normalization of $\rho_{s}$, we find that the solution to Eq. (29) is given by

$$
\rho_{s}(\theta)=\frac{1}{2 \pi}\left[1+d_{s} \cos j_{s}\left(\theta-\theta_{0 s}\right)\right]
$$

where $j_{s}=1,2, \cdots$. Substituting this solution back into Eq. (29), we find

$$
\bar{\mu}_{s}=\frac{j_{s}^{2}}{4}, \quad V_{s}=-\frac{j_{s}^{2}}{4 \pi}
$$

and

$$
W_{s}^{2}=\frac{j_{s}^{2}}{4 \pi^{2}}\left(1-d_{s}^{2}\right)
$$

The undetermined constants $d_{s}, j_{s}$ and $\theta_{0 s}$ are finally determined by inserting Eq (30) into Eq. (23). For consistency, we require $\theta_{0 A}=\theta_{0 B}=\theta_{0}, j_{A}=j_{B}=j$ and

$$
\frac{d_{B}}{d_{A}}=r=-\frac{x_{A}}{x_{B}} .
$$

In view of the arbitrary phase angle $\theta_{0}$, we can without loss of generality choose $d_{B}$ to be positive.

Once the densities are known, the phases can be determined from Eq. (13). An angular integration of this equation leads to the general phase-density relation

$$
\varphi_{s}(\theta)-\varphi_{s}\left(\theta_{0}\right)=\frac{\Omega}{2}\left(\theta-\theta_{0}\right)+\frac{W_{s}}{2} \int_{\theta_{0}}^{\theta} \frac{d \theta^{\prime}}{\rho_{s}\left(\theta^{\prime}\right)} .
$$


Using Eq. (30) and writing $j\left(\theta-\theta_{0}\right)=n \pi+\bar{\theta}$, where $n$ is a non-negative integer and $0 \leq \bar{\theta}<\pi$, one finds

$$
\begin{aligned}
& \varphi_{s}(\theta)-\varphi_{s}\left(\theta_{0}\right)-\frac{\Omega}{2}\left(\theta-\theta_{0}\right) \\
= & \frac{\operatorname{sgn} W_{s}}{2}\left\{n \pi+2 \tan ^{-1}\left[\left(\frac{1+d_{s}}{1-d_{s}}\right)^{ \pm \frac{1}{2}} \tan \frac{\bar{\theta}}{2}\right]\right\},
\end{aligned}
$$

where the $+(-)$ sign corresponds to even (odd) $n$ values. Finally, to evaluate the phase boundary condition in Eq. (10), we insert $\theta-\theta_{0}=2 \pi$ (which implies $n=2 j$ and $\bar{\theta}=0$ ) in Eq. (35). This gives immediately

$$
\Omega=2 J_{s}-j \operatorname{sgn} W_{s},
$$

which determines the value of $\Omega$. We see that the angular velocity for these solutions is a constant, independent of the parameter $d_{s}$ which specifies a particular solution. Eliminating $\Omega$ from Eq. (36) we find

$$
J_{B}-J_{A}=\frac{j}{2}\left(\operatorname{sgn} W_{B}-\operatorname{sgn} W_{A}\right)
$$

for the solutions given by Eq. (30) and Eq. (35).

\section{Class (ii) solutions: $c_{A} x_{A}+c_{B} x_{B} \neq 0$}

In this case Eq. (19) admits the following soliton train solutions [5, 12, 13]

$$
\rho_{s}(\theta)=\mathcal{N}_{s}\left(\eta_{s}\right)\left[1+\eta_{s} \operatorname{dn}^{2}\left(\frac{j_{s} K}{\pi}\left(\theta-\theta_{0 s}\right) \mid m_{s}\right)\right],
$$

where $\operatorname{dn}(u \mid m)$ is a Jacobi elliptic function [16] and $j_{s}=$ $1,2, \cdots$. Here

$$
\eta_{s}=\frac{-2 j_{s}^{2} K^{2}\left(m_{s}\right)}{g_{s}}, \quad \mathcal{N}_{s}\left(\eta_{s}\right)=\frac{g_{s}}{2 \pi^{3} \gamma_{s}},
$$

and

$$
g_{s} \equiv \pi^{2} \gamma_{s}+2 j_{s}^{2} K\left(m_{s}\right) E\left(m_{s}\right),
$$

where $K(m)$ and $E(m)$ are, respectively, complete elliptic integrals of the first and second kind with elliptic parameter $m$ [16]. We point out that this form of solution applies to both bright (attractive interactions) and grey/dark (repulsive interactions) solitons. In addition, these single-species solitons are completely specified by the elliptic parameter $m_{s}$, the interaction parameter $\gamma_{s}$ and the soliton train index $j_{s}$. Substituting the solutions in Eq. (38) back into Eq. (19), one finds [5]

$$
\bar{\mu}_{s}=\frac{3 g_{s}}{2 \pi^{2}}-\frac{\left(2-m_{s}\right) j_{s}^{2} K^{2}\left(m_{s}\right)}{\pi^{2}} .
$$

and

$$
W_{s}^{2}=\frac{g_{s} f_{s} h_{s}}{2 \pi^{8} \gamma_{s}^{2}}
$$

where $f_{s} \equiv g_{s}-2 j_{s}^{2} K^{2}\left(m_{s}\right)$ and $h_{s} \equiv g_{s}-2(1-$ $\left.m_{s}\right) j_{s}^{2} K^{2}\left(m_{s}\right)$.

We turn next to the implications of the density ansatz in Eq. (23). Substituting Eq. (38) into Eq. (23), we observe first that consistency requires $\theta_{0 A}=\theta_{0 B}=\theta_{0}$, $m_{A}=m_{B}=m$ and $j_{A}=j_{B}=j$. More remarkably, one can check that Eq. (23) is satisfied identically for any value of the parameter $r$ provided the interaction parameters are related by Eq. (20). Thus the pair of soliton train solutions in Eq. (38) are indeed solutions to Eq. (16). To specify these solutions completely, however, we must still determine the parameters $r$ and $m$.

To do so we must next make use of the phase boundary conditions in Eq. (10). Inserting the densities in Eq. (38) into Eq. (34), one finds that the phases are given by

$$
\varphi_{s}(\theta)-\varphi_{s}\left(\theta_{0}\right)=\frac{\Omega}{2}\left(\theta-\theta_{0}\right)+\frac{\pi^{4} \gamma W_{s}}{j f_{s} K} \Pi\left(n_{s} ; u \mid m\right),
$$

where $n_{s}=-2 j^{2} m K^{2} / f_{s}, u=j K\left(\theta-\theta_{0}\right) / \pi$ and $\Pi\left(n_{s} ; u \mid m\right)$ is the elliptic integral of the third kind. The phase boundary conditions then yield

$$
\Omega=2 J_{s}-2 \mathcal{M}_{s} \operatorname{sgn} W_{s},
$$

where $\mathcal{M}_{s}=\sqrt{2 g_{s} h_{s} / f_{s}} \Pi\left(n_{s} ; K \mid m\right) /(2 \pi K)$. Eliminating $\Omega$ from Eq. (44) we obtain

$$
J_{B}-J_{A}=\mathcal{M}_{B} \operatorname{sgn} W_{B}-\mathcal{M}_{A} \operatorname{sgn} W_{A} .
$$

This is the key equation that determines $r=c_{B} / c_{A}$ as a function of the elliptic parameter $m$ for this class of solutions. In other words, once the relationship between $r$ and $m$ is established, the solutions of physical interest are generated by allowing $m$ to vary over some well-defined interval to be determined.

\section{MEAN-FIELD YRAST SPECTRUM}

In this section, we use the phase boundary conditions, Eq. (37) and Eq. (45), to determine the mean-field yrast spectrum $\bar{E}_{0}(l)$. As shown in [10, 11] it is advantageous to consider the energy $\bar{e}_{0}(l) \equiv \bar{E}_{0}-l^{2}$, which is a periodic function of the angular momentum per particle $l$, with unit period. In addition, $\bar{e}_{0}(l)$ has the inversion property $\bar{e}_{0}(-l)=\bar{e}_{0}(l)$, which reflects the fact that the energy does not depend on the sense of the angular momentum. It is thus sufficient to restrict our discussion of the yrast spectrum to $0 \leq l \leq 1 / 2$. Finally, as the soliton solutions with $j>1$ generally have higher energies, we will focus on the case of $j=1$, namely the single-soliton states.

Before we consider the two classes of solutions individually, it is useful to observe that both phase boundary conditions, Eqs. (37) and (45), involve only the difference of the phase winding numbers $\mathcal{J} \equiv J_{B}-J_{A}$. From Eq. (36) we see that a simultaneous shift of both $J_{A}$ and $J_{B}$ by an integer $p$ simply changes the angular velocity 
from $\Omega$ to $\Omega+2 p$ and, in view of Eqs. (14) and (15), the angular momentum from $l$ to $l+p$. In addition, a simultaneous sign reversal of $J_{A}$ and $J_{B}$, along with that of $W_{A}$ and $W_{B}$, changes the angular velocity from $\Omega$ to $-\Omega$ and the angular momentum from $l$ to $-l$.

We now consider the class of solutions obtained for $c_{A} x_{A}+c_{B} x_{B}=0$, given by Eq. (30) and Eq. (35). These solutions will be shown to represent those states for which the angular momentum per particle lies in the range $0 \leq$ $l \leq x_{B}$ (in the interval $0 \leq l \leq 1 / 2$ of interest). To begin, we observe from the boundary condition in Eq. (37), that $\mathcal{J}=J_{B}-J_{A}$ can only take the value 0 and \pm 1 for $j=1$. We find that the following two combinations of $J_{A}$ and $J_{B}$ yield angular momenta that lie within $0 \leq l \leq 1 / 2$ : (i) $J_{A}=J_{B}=0$ with $\operatorname{sgn} W_{\mathrm{A}}=\operatorname{sgnW}_{\mathrm{B}}=-1$; (ii) $J_{A}=0$ and $J_{B}=1$ with $\operatorname{sgn} W_{A}=-1$ and $\operatorname{sgn} W_{B}=1$. From Eq. (36) we find that $\Omega=1$ for both (i) and (ii).

A combination of Eqs. (15), (32) and (36) shows that the angular momentum of each species can be expressed as

$$
l_{s}=J_{s}+\frac{1}{2} \operatorname{sgn} W_{s}\left(\sqrt{1-d_{s}^{2}}-1\right) .
$$

For $J_{s}=0$ and $d_{s}=0$ we have a uniform density state of zero angular momentum. In order for $l_{s}$ to increase to positive values as $\left|d_{s}\right|$ increases from zero, we must choose $\operatorname{sgn} W_{s}$ to be negative. This establishes parameter set (i). Since $\left|d_{B}\right|>\left|d_{A}\right|$, the allowable range of $\left|d_{B}\right|$ values is set by the requirement that $W_{B}^{2}$ is non-negative. This determines that $0 \leq\left|d_{B}\right| \leq 1$. As $\left|d_{B}\right|$ increases continuously from 0 to 1 , Eq. (46) shows that $l_{A}$ increases from 0 to $\left(x_{A}-\sqrt{x_{A}-x_{B}}\right) / 2 x_{A}$ and $l_{B}$ increases from 0 to $1 / 2$. The total angular momentum per particle as a result increases from zero to $\left(1-\sqrt{x_{A}-x_{B}}\right) / 2$. At this point, the density of the $B$ component acquires a node at $\theta=\theta_{0}+\pi$ (recall that $d_{B}$ is chosen to be positive) and the phase winding number $J_{B}$ jumps by 1 as $l$ increases further. By the same token $\operatorname{sgn} W_{B}$ must become positive in order that $l_{B}$ be continuous with further variations of $\left|d_{B}\right|$. On the other hand $J_{A}$ and $\operatorname{sgn} W_{A}$ retain their values of 0 and -1 , respectively, thus defining parameter set (ii). As $\left|d_{B}\right|$ is reduced continuously back to zero, $l_{A}$ decreases from $\left(x_{A}-\sqrt{x_{A}-x_{B}}\right) / 2 x_{A}$ to zero while $l_{B}$ continues to increases from $1 / 2$ to 1 , at which point all the circulation is carried by the $B$ component and the total angular momentum is $l=x_{B}$. Using Eq. (35), we evaluate the phases of the components at this point and find $\varphi_{A}(\theta)-\varphi_{A}\left(\theta_{0}\right)=0$ and $\varphi_{B}(\theta)-\varphi_{B}\left(\theta_{0}\right)=\theta-\theta_{0}$. This shows that the components are in the plane wave states $\psi_{A}=1 / \sqrt{2 \pi}$ and $\psi_{B}=e^{i\left(\theta-\theta_{0}\right)} / \sqrt{2 \pi}$ when $l=$ $x_{B}$.
To obtain the energy spectrum for the states given by Eq. (30) and Eq. (35), we can use the formula given in Eq. (24). However, a simpler way in this case is to make use of the fact that $\Omega=1$ and integrate Eq. (7). Recalling that the ground state energy in the mean-field theory is $\gamma / 2$, we find

$$
\bar{E}_{0}=\frac{\gamma}{2}+l
$$

for $0 \leq l \leq x_{B}$, which coincides with the yrast spectrum of an ideal gas. It can be shown 10, 11] that the solutions in Eq. (301) and Eq. (35) in fact yield the lowest possible mean-field energy as a function of $l$. In other words, Eq. (47) indeed gives the yrast spectrum for $0 \leq l \leq x_{B}$.

The states with angular momenta within the range $x_{B} \leq l \leq 1 / 2$ belong to the second class of soliton solutions, given by Eq. (38) and Eq. (43). We again focus on the $j=1$ case and consider the boundary condition in Eq. (45), which determines $r$ as a function of the elliptic parameter $m$. Once $r(m)$ is determined, various physical quantities can then be evaluated using the formulae given in Sec. II. Unlike Eq. (37) for the first class of solutions, however, Eq. (45) can only be solved numerically and generally admits solutions for $\mathcal{J}>1$ even in the case of $j=1$. Furthermore, the solution $r=r(m, \mathcal{J})$ is multi-branched for fixed values of $\mathcal{J}$, adding to the complexity of the problem. We will focus on branches with negative values of $r(m, \mathcal{J})$ (grey-bright solitons), as these yield lower energies than the positive branches (grey-grey solitons).

For orientation purposes, it is useful to first determine $r_{0}(\mathcal{J}) \equiv r(m=0, \mathcal{J})$. It is important to note that, should $m=0$ solutions exist, Eq. (38) and Eq. (43) imply that the $A$ and $B$ components are both in plane wave states. More specifically, one obtains at these $r$ values the wave functions $\psi_{A}=e^{i J_{A}\left(\theta-\theta_{0}\right)} / \sqrt{2 \pi}$ and $\psi_{B}=e^{i J_{B}\left(\theta-\theta_{0}\right)} / \sqrt{2 \pi}$, for which the total angular momentum is $l=x_{A} J_{A}+x_{B} J_{B}$. Thus, to obtain solutions whose angular momenta are within $x_{B} \leq l \leq 1 / 2, J_{A}$ clearly has to be zero while $J_{B}=\mathcal{J}$ can take on various positive integer values dependening on the size of $x_{B}$.

Setting $m=0$ in Eq. (45), we find the simplified equation (see the Appendix for the calculation of $\mathcal{M}_{s}$ )

$$
\mathcal{J}=\frac{1}{2} \operatorname{sgn} W_{B} \sqrt{2 \gamma_{B}+1}-\frac{1}{2} \operatorname{sgn} W_{A} \sqrt{2 \gamma_{A}+1} .
$$

Using the expressions for $\gamma_{s}$ in Eq. (20), this equation can be put into the form of the following quartic equation for $r_{0}(\mathcal{J})$

$$
\gamma^{2}\left(x_{B} r_{0}+x_{A}\right)^{2}\left(r_{0}-1\right)^{2}-4 \mathcal{J}^{2} \gamma r_{0}\left(r_{0}+1\right)\left(x_{B} r_{0}+x_{A}\right)+4 \mathcal{J}^{2}\left(\mathcal{J}^{2}-1\right) r_{0}^{2}=0 .
$$

The number of negative solutions that this equation admits depends on the values of $\gamma, x_{B}$ and $\mathcal{J}$. In view of 
our earlier discussion, the case of $\mathcal{J}=0$ is not of interest when $l>x_{B}$. For $\mathcal{J}=1$, it is not difficult to see that there are always two negative solutions, one of which is $r_{0}=-x_{A} / x_{B}$ for arbitrary $\gamma$ and $x_{B}$. This special root for $\mathcal{J}=1$ is associated with the class (i) solutions considered earlier. The other corresponds to a different soliton branch that begins when $l$ exceeds $x_{B}$. The situation

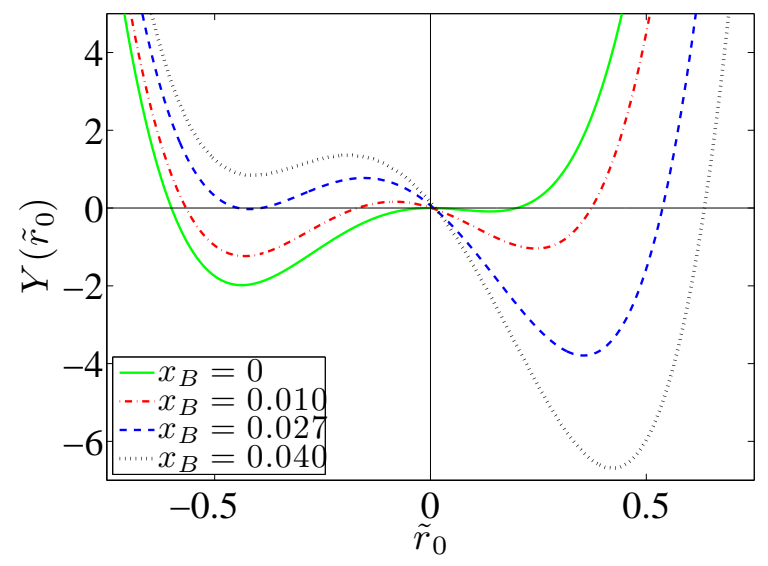

FIG. 1: The quartic function $Y\left(\tilde{r}_{0}\right)$ plotted for various $x_{B}$ values for $\gamma=10$ and $\mathcal{J}=2$.

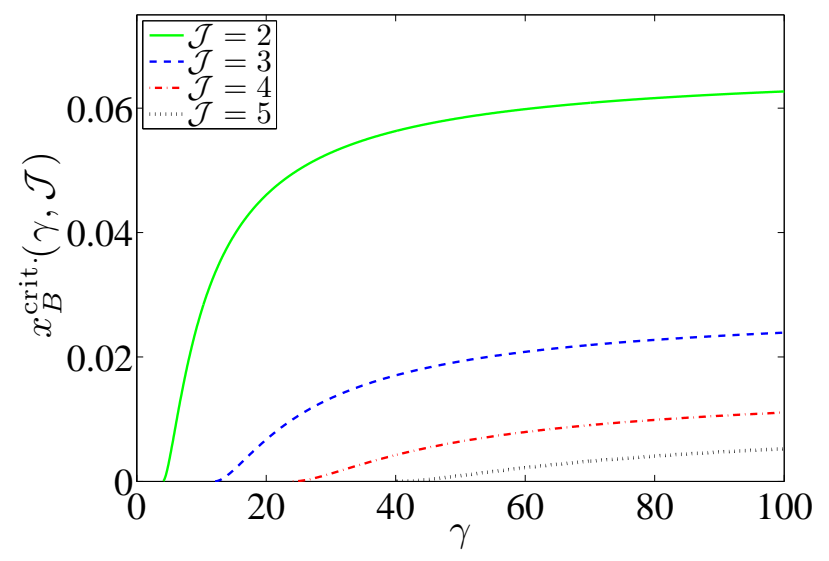

FIG. 2: The critical value $x_{B}^{\text {crit }}(\gamma, \mathcal{J})$ plotted as a function of $\gamma$ for $\mathcal{J}=2$ (solid, green), 3 (dashed, blue), 4 (dot-dash, red) and 5 (dotted, black).

for $\mathcal{J}>1$ is slightly more complicated. To analyze it, we multiply both sides of Eq. (49) by $x_{B}^{2}$ and rewrite it as an equation for $\tilde{r}_{0}=x_{B} r_{0}$

$$
Y\left(\tilde{r}_{0}\right) \equiv y\left(\tilde{r}_{0}\right)+z\left(\tilde{r}_{0}\right)=0,
$$

where

$$
y\left(\tilde{r}_{0}\right)=\tilde{r}_{0}^{2}\left[\gamma \tilde{r}_{0}+\gamma-2 \mathcal{J}(\mathcal{J}-1)\right]\left[\gamma \tilde{r}_{0}+\gamma-2 \mathcal{J}(\mathcal{J}+1)\right]
$$

and

$$
\begin{aligned}
z\left(\tilde{r}_{0}\right)= & -4 x_{B} \gamma^{2} \tilde{r}_{0}^{3}-6 x_{A} x_{B} \gamma^{2} \tilde{r}_{0}^{2} \\
& +2 x_{A} x_{B} \gamma\left[\left(x_{B}-x_{A}\right) \gamma-\mathcal{J}^{2}\right] \tilde{r}_{0}+\gamma^{2} x_{A}^{2} x_{B}^{2}
\end{aligned}
$$

The cubic $z\left(\tilde{r}_{0}\right)$ is proportional to $x_{B}$ and thus does not influence the locations of the roots given by $y\left(\tilde{r}_{0}\right)=0$ in the $x_{B} \rightarrow 0$ limit. The quartic $y\left(\tilde{r}_{0}\right)$ has a double root at $\tilde{r}_{0}=0$ and two single roots at $[-\gamma+2 \mathcal{J}(\mathcal{J} \pm 1)] / \gamma$. It is clear that it has two negative roots if $\gamma>2 \mathcal{J}(\mathcal{J}+1)$, which guarantees that $Y\left(\tilde{r}_{0}\right)$ has two negative roots for sufficiently small $x_{B}$. This condition, however, is actually too strong. If $2 \mathcal{J}(\mathcal{J}-1)<\gamma<2 \mathcal{J}(\mathcal{J}+1), y\left(\tilde{r}_{0}\right)$ has one positive and one negative root (Fig. 1 gives a numerical example for $\mathcal{J}=2$ and $\gamma=10$ ). As $x_{B}$ is increased from 0 , the cubic $z\left(\tilde{r}_{0}\right)$ removes the root degeneracy at $\tilde{r}_{0}=0$ and two negative roots of $Y\left(\tilde{r}_{0}\right)$ appear.

We thus find that for any $\mathcal{J}>1$, negative solutions of Eq. (49) (always two) exist if $\gamma>2 \mathcal{J}(\mathcal{J}-1)$ and if $x_{B}$ is sufficiently small. As $x_{B}$ increases further, the two negative roots approach each other and a double negative root eventually appears at some critical value $x_{B}^{\text {crit }}(\gamma, \mathcal{J})$, above which no negative root is possible. This behaviour is again illustrated in Fig. 1, The condition for the existence of a double negative root is given by

$$
\Delta=0,
$$

where $\Delta$ is the discriminant [14] of $Y\left(\tilde{r}_{0}\right)$. The discriminant $\Delta$ is a polynomial function of $x_{B}$ and $\gamma$; since it is rather unwieldy, we will not write it out here. It is nonetheless straightforward to solve Eq. (53) numerically. The results obtained for $x_{B}^{\text {crit }}(\gamma, \mathcal{J})$ as a function of $\gamma$ for $\mathcal{J}=2,3,4$ and 5 are shown in Fig. 2. Each of these curves begins at $\gamma=2 \mathcal{J}(\mathcal{J}-1)$ and below this value, a double negative root for that particular value of $\mathcal{J}$ cannot exist for any value of $x_{B}$. It is convenient to extend the definition of $x_{B}^{\text {crit }}(\gamma, \mathcal{J})$ by defining $x_{B}^{\text {crit }}(\gamma, \mathcal{J})=0$ for $\gamma<2 \mathcal{J}(\mathcal{J}-1)$. In this way, we can say quite generally that negative roots do not exist if $x_{B}>x_{B}^{\text {crit }}(\gamma, \mathcal{J})$. Since two negative solutions always exist for $\mathcal{J}=1$, we can simply define $x_{B}^{\text {crit }}(\gamma, 1)=1 / 2$ for all $\gamma$, where $x_{B}=1 / 2$ is the maximum value of the minority concentration. Our calculations show that, for a fixed $\gamma, x_{B}^{\text {crit }}(\gamma, \mathcal{J})$ diminishes rapidly with increasing $\mathcal{J}$. Furthermore, $x_{B}^{\text {crit }}(\gamma, \mathcal{J})$ increases monotonically as a function of $\gamma$ and approaches an asymptotic value as $\gamma \rightarrow \infty$. An analysis of Eq. (53) in the $\gamma \rightarrow \infty$ limit yields the result

$$
\lim _{\gamma \rightarrow \infty} x_{B}^{\text {crit }}(\gamma, \mathcal{J})=\frac{1}{2}-\frac{1}{2} \sqrt{1-\mathcal{J}^{-2}}
$$

Fig. 2 is extremely useful in determining what the possible negative roots of Eq. (49) are. We imagine 

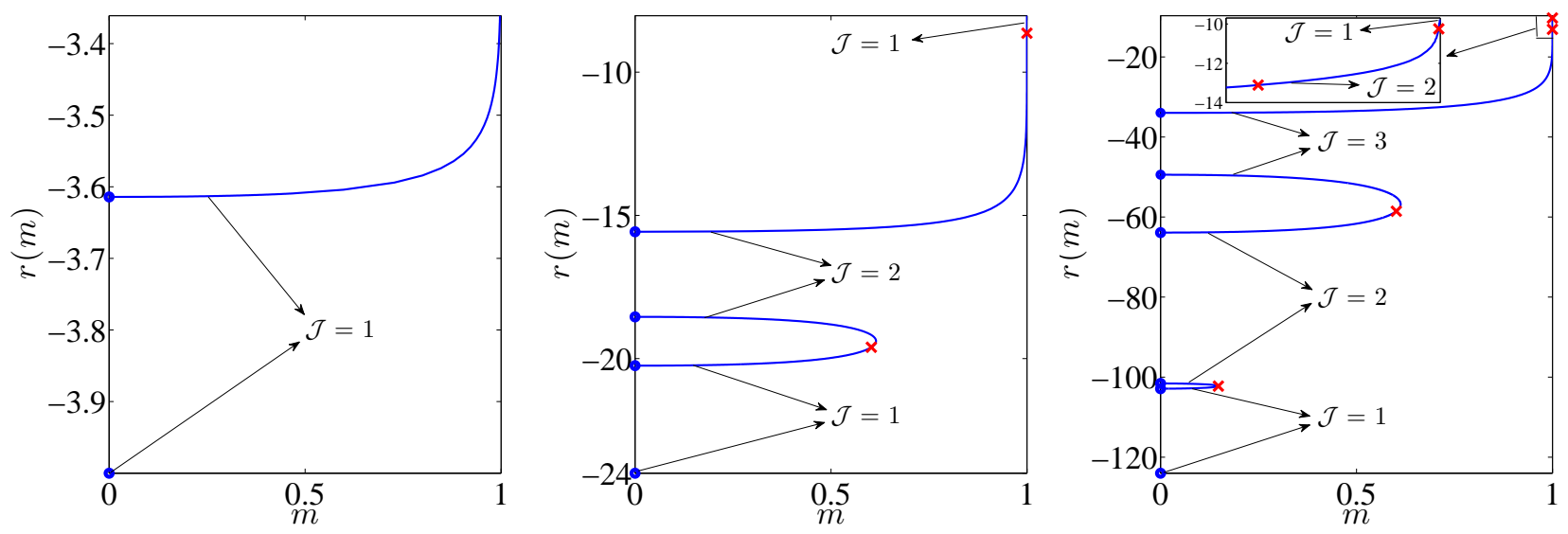

FIG. 3: The ratio $r(m)$ plotted as a function of $m$ for $x_{B}=0.2$ (left), 0.04 (middle) and 0.008 (right). The dots on the $r$ axis mark the values of $r_{0}(\mathcal{J})$ and the crosses mark the location where the phase winding number $J_{B}$ has a jump of \pm 1 . The inset in the right figure is an expanded view of the $r(m)$ curve in the vicinity of $m=1$. The interaction parameter is $\gamma=23$.

drawing a vertical line in Fig. 2 at some particular $\gamma$ value. This line will intersect the $x_{B}^{\text {crit }}(\gamma, \mathcal{J})$ curves for $\mathcal{J} \leq \mathcal{J}_{\max }$. For a given choice of $x_{B}$, negative roots occur if $x_{B}<x_{B}^{\text {crit }}(\gamma, \mathcal{J})$, with $\mathcal{J} \leq \mathcal{J}_{\text {max }}$. This condition will be satisfied for some maximum winding number $q \leq \mathcal{J}_{\max }$. In other words, negative $r_{0}(\mathcal{J})$ roots will appear for $\mathcal{J}=1, \ldots, q$. We denote these pairs of roots by $r_{0}^{<}(\mathcal{J})$ and $r_{0}^{>}(\mathcal{J})$, with $r_{0}^{<}(\mathcal{J})<r_{0}^{>}(\mathcal{J})$. In addition, we find that $r_{0}^{>}(\mathcal{J})<r_{0}^{<}(\mathcal{J}+1)$, so that the roots form an increasing sequence which can be plotted along the $m=0$ axis in the $r(m)-m$ plane. An example of this is shown in Fig. 3. The roots in this sequence are bounded from below by $r_{0}^{<}(1)=-x_{A} / x_{B}$.

The above information can now be used to numerically determine the negative $r(m)$ solutions of Eq. (45) for $m \neq$ 0 . We find that there exists a unique value of $m$ for each $r$ value in the range $r_{0}^{>}(\mathcal{J}) \leq r \leq r_{0}^{<}(\mathcal{J}+1)$ for $\mathcal{J}=1, \cdots, q-1$ and $r_{0}^{>}(q) \leq r \leq r_{0, \max }$, where $r_{0, \max }$ is an upper bound whose value depends on $\gamma$ and $x_{B}$; no solution exists for negative $r$ outside one of these ranges. When plotted in the $r(m)-m$ plane, the solutions quite generally either form a lobe which starts and ends on the $m=0$ axis or a curve which extends from $m=0$ to $m=m_{\max }$. The number of lobes is given by $q-1$. This behaviour is illustrated in Fig. 3 for the example of $\gamma=23$, in which case $\mathcal{J}_{\max }=3$.

In the left panel of Fig. $3\left(x_{B}=0.2\right), q=1$ and no lobe appears in the solution of $r(m)$; the phase winding number difference is fixed at $\mathcal{J}=1$. The isolated point at $r=-x_{A} / x_{B}$ on the $m=0$ axis corresponds to the $l \rightarrow$ $x_{B}$ limit of the class (i) solution which, as we have seen, is $\psi_{A}=1 / \sqrt{2 \pi}$ and $\psi_{B}=e^{i\left(\theta-\theta_{0}\right)} / \sqrt{2 \pi}$. The continuous curve is a different soliton branch which at $m=0$ reduces to exactly the same plane wave solution with $l=x_{B}$. As $m$ increases, $l$ increases continuously from $x_{B}$ to $1 / 2$. Since $r(m)>r_{0}^{<}(1)$, we have $\gamma_{A}>0$ and $\gamma_{B}<0$, which implies that the $A$ component forms a grey soliton while the $B$ component forms a bright soliton.

More generally for $q>1$, as illustrated in the middle and right panels of Fig. 3 the two $r_{0}(\mathcal{J})$ negative roots correspond to the plane wave state $\psi_{A}=1 / \sqrt{2 \pi}$ and $\psi_{B}=e^{i \mathcal{J}\left(\theta-\theta_{0}\right)} / \sqrt{2 \pi}$ with $\mathcal{J}=1, \ldots, q$. Each branch of the $r$ vs $m$ curves, labelled from the bottom to the top by the index $k=1, \ldots, q$, represents a distinct soliton state. On the $k$-th lobe $(k \leq q-1)$, the angular momentum increases from $k x_{B}$ at the lower end to $(k+1) x_{B}$ at the upper end. On the final $k=q$ branch, the angular momentum continues to increase from $l=q x_{B}$ to $l=1 / 2$. We emphasize that the disconnected structure of the $r(m)$ branches does not imply any discontinuous behaviour of the condensate wave functions as a function of $l$. In fact, since $r_{0}^{<}(\mathcal{J})$ and $r_{0}^{>}(\mathcal{J})$ correspond to the same plane wave functions, $\psi_{s}=e^{i J_{s}\left(\theta-\theta_{0}\right)}$, the $m=0$ wave functions at the top of one branch are the same as those at the bottom of the next. In other words, the various soliton branches are always stitched together by plane wave functions at angular momenta which are multiples of $x_{B}$.

We also find that the phase winding number $J_{B}$ exhibits a rather interesting behaviour as a function of $l$. Since the plane wave state of the $B$ component at the end-points of the $k$-th lobe changes from $\psi_{B}=$ $e^{i k\left(\theta-\theta_{0}\right)} / \sqrt{2 \pi}$ to $\psi_{B}=e^{i(k+1)\left(\theta-\theta_{0}\right)} / \sqrt{2 \pi}$, the phase winding number $J_{B}$ must increase by 1 along this path. This means that $J_{B}$ must jump from $k$ to $k+1$ somewhere along the path. This behaviour is confirmed by our numerical calculations which show that the jump in $J_{B}$ occurs at the point along the path where the $B$-component density develops a node at $\theta=\theta_{0}+\pi$, that is, opposite to where its density is a maximum. These points along the path are indicated by the red crosses in Fig. $3 \mathrm{Fi}-$ nally, on the $k=q$ branch, $J_{B}$ decreases from $q$ to 1 via 

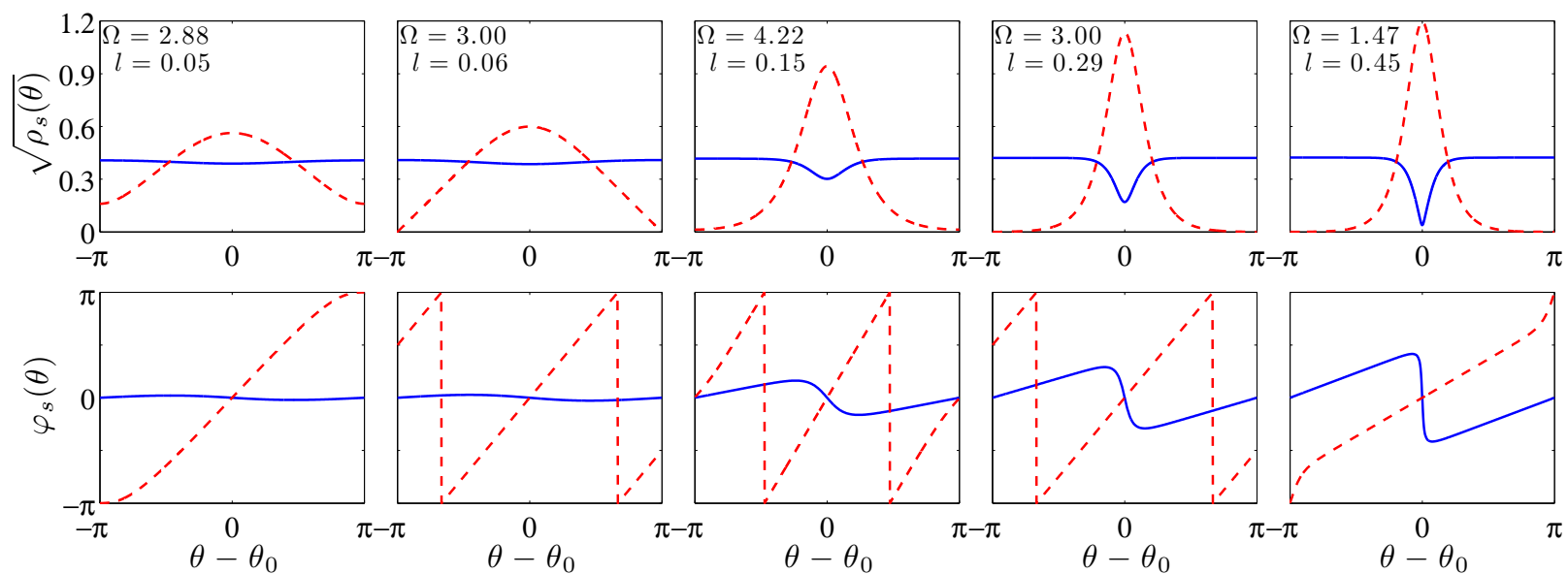

FIG. 4: Soliton amplitude and phase at various angular momenta for $x_{B}=0.04$ and $\gamma=23$. The solid (blue) and dashed (red) lines represent the $A$ and $B$ components, respectively.

a sequence of $\theta=\theta_{0}+\pi$ nodes. Thus, the phase of the $B$ component first winds up for $k=1, \ldots, q-1$ and then unwinds on the $k=q$ branch as the angular momentum per particle $l$ varies continuously from $x_{B}$ to $1 / 2$. These features can be seen clearly from the density and phase plots of the solitons at various angular momenta, shown in Fig. 4 for $x_{B}=0.04$. The figure shows that $J_{A}=0$ over this range of angular momenta while $J_{B}$ starts at 1 , jumps to 2 at $l=0.06$ and then back to 1 at $l=0.29$. At $l=1 / 2$, both components develop a node and $J_{A}$ jumps from 0 to 1 while $J_{B}$ jumps from 1 to 0 .

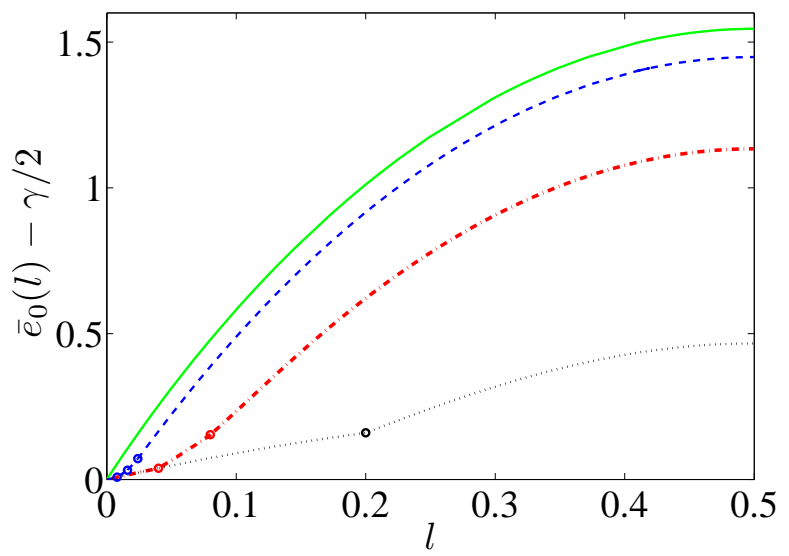

FIG. 5: The yrast spectrum $\bar{e}_{0}(l)$ as a function of $l$ plotted for various values of $x_{B}$. The curves from bottom to the top correspond to, $x_{B}=0.2$ (dot, black), 0.04 (dot-dash, red), 0.008 (dash, blue), and 0 (solid, green). The circles mark the locations at which the slope of the curves has a discontinuity (see Figure 6). The interaction parameter is $\gamma=23$.

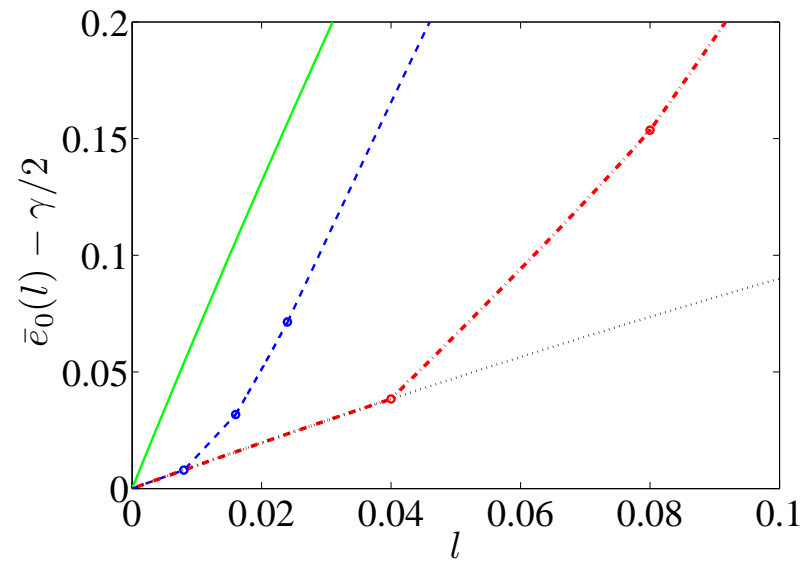

FIG. 6: An expanded view of the small $l$ region of Fig. 5

We now use the solution of $r(m)$ for the grey-bright solitons to evaluate the mean-field yrast spectrum from Eq. (24). In Fig. (5) we plot $\bar{e}_{0}(l)-\gamma / 2$ for $\gamma=23$ and various concentrations, $x_{B}$. We see that the yrast spectrum is a continuous function of $l$ but consists of several segments corresponding to the different soliton states that occur. The first segment for $0 \leq l \leq x_{B}$ is the energy of the class (i) solution, which is given by $\bar{e}_{0}(l)=l-l^{2}+\gamma / 2$ (see Eq. (47)). The remaining segments correspond to the distinct $r(m)$ branches in Fig. 3, Generally speaking, there are $q-1$ segments, each of width $x_{B}$, associated with the lobes in Fig. 3 and a final segment extending from $l=q x_{B}$ to $l=1 / 2$. Although the energy $\bar{e}_{0}(l)$ is a continuous function of $l$, its derivative, which is related to the angular velocity $\Omega$ through $d \bar{e}_{0}(l) / d l=\Omega-l$, is 
not. The discontinuities occur at $l=k x_{B}(k=1, \ldots, q)$, the end-points of the various segments. As a result, the angular velocity $\Omega$ has different limiting values as the angular momentum approaches one of these points from either side. More quantitatively, using Eq. (44) we find that the two angular velocity limits at $l=k x_{B}$ are

$$
\Omega_{k}^{\gtrless}=\sqrt{1+2 \gamma_{A}^{\gtrless}(k)},
$$

where $\gamma_{A}^{\gtrless}(k)=\gamma\left[x_{A}+x_{B} r_{0}^{\gtrless}(k)\right]$ and $<(>)$ denotes the limit as $l$ approaches $k x_{B}$ from lesser (greater) values. In deriving this result we made use of the fact that $\operatorname{sgn} W_{A}=$ -1 in Eq. (44). To see this, we observe that $\mathcal{M}_{A} \geq$ $\mathcal{M}_{B}>0$ for $\gamma_{A}>0$ and $\gamma_{B}<0$ (see Appendix). Using this property in Eq. (45) and bearing in mind that $\mathcal{J}=$ $J_{B}-J_{A}>0$, we find $\operatorname{sgn} W_{A}=-1$. From Eq. (55) we also find the following inequalities

$$
1 \leq \Omega_{k-1}^{<} \leq \Omega_{k-1}^{>} \leq \Omega_{k}^{<} \leq \Omega_{k}^{>}
$$

which will be useful in the discussion of persistent currents in the next section.

\section{PERSISTENT CURRENT AT HIGHER ANGULAR MOMENTUM}

In this section, we investigate within mean-field theory the possibility of persistent currents of the twocomponent system at higher angular momenta. For a single component repulsive gas (the $x_{B}=0$ limit of the two-component system), persistent currents are sustainable at any integer angular momentum $l=\nu$ provided the interaction strength $\gamma$ exceeds a critical value given by [11]

$$
\gamma=\frac{4 \nu^{2}-1}{2}
$$

Although the addition of a second component has the tendency of destabilizing persistent currents at higher angular momenta, it does not completely remove the possibility, contary to the suggestion made in Ref. [10]. In support of this we presented in an earlier paper [11] a general argument based on the idea that the energy spectrum of the two-component system should continuously reduce to the single component result as the concentration of the minority component $x_{B}$ gradually goes to zero. The purpose of this section is to use the exact soliton solutions for the yrast spectrum to provide a more definitive, and quantitative, argument for why persistent currents at higher angular momenta are indeed possible.

The possibility of persistent currents is predicated on the existence of local minima in the yrast spectrum. For a single component gas, local minima (if they exist) always occur at integer angular momenta per particle. This is no longer the case for a two-component system. In fact, as a result of the non-interacting-like spectrum for $0 \leq l \leq$
$x_{B}$, local minima of the yrast spectrum cannot appear at integer values of angular momentum $l$. However, as a key result of this section, we will demonstrate that local minimum can form at angular momenta $l=\nu x_{A}=$ $\nu\left(1-x_{B}\right)$ for any integer $\nu$, provided that $\gamma$ is greater than the critical strength

$$
\gamma_{c r, \nu}=\frac{4 \nu^{2}-1}{2\left(1-4 x_{B} \nu^{2}\right)} .
$$

This proves unequivocally that persistent currents are possible at arbitrarily high angular momentum. We observe that in the $x_{B} \rightarrow 0$ limit, $\gamma_{c r, \nu}$ in fact reduces to the single-component critical criterion given in Eq. (57). A corollary of Eq. (58) is that a local minimum at $l=\nu x_{A}$ cannot arise for any $\gamma$ if

$$
x_{B}>\frac{1}{4 \nu^{2}} .
$$

To derive the result in Eq. (58), we focus on the angular velocity of the solitons as a function of $l$ which is equal to the slope of the mean-field yrast spectrum $\bar{E}_{0}(l)$. We will be particularly interested in parameter regimes where the angular velocity develops discontinuities at $l=k x_{B}$. From the periodicity of the yrast spectrum, the angular velocity will also have discontinuities at $l=\nu-k x_{B}$. Our objective is to investigate the possibility of the formation of a local minimum at one of these angular momenta. The condition for this to occur is that the right limit of the angular velocity is positive and the left limit is negative.

In the argument to be presented, we require the index $k$ to span a range that includes the integer $\nu$. To ensure this, we assume that $\gamma>2 \nu(\nu-1)$ and that $x_{B}<x_{B}^{\text {crit }}(\gamma, \nu)$ so that the number of discontinuities is $q \geq \nu$. Due to the inversion symmetry and periodicity of the yrast spectrum, the angular velocity at $\nu-l$ is related to that at $l$ through

$$
\Omega(\nu-l)=2 \nu-\Omega(l) .
$$

This implies that the left limit of the angular velocity at $l=\nu-k x_{B}$ is

$$
\Omega^{<}\left(\nu-k x_{B}\right)=2 \nu-\Omega_{k}^{>},
$$

while the right limit is

$$
\Omega^{>}\left(\nu-k x_{B}\right)=2 \nu-\Omega_{k}^{<},
$$

where $\Omega_{k}^{\gtrless}$ is given in Eq. (55)). In view of Eq. (56), we see that $\Omega^{<}\left(\nu-(k+1) x_{B}\right)<\Omega^{<}\left(\nu-k x_{B}\right)$, that is, the left limit decreases with increasing $k$. We will prove that $\Omega^{<}\left(\nu-k x_{B}\right)$ is positive for $k<\nu$ and that $\Omega^{<}\left(\nu-\nu x_{B}\right)$ is the first term in the sequence which can become negative with increasing $\gamma$. This occurs when $\Omega_{\nu}^{>}>2 \nu$. We thus focus on $\Omega_{\nu}^{\gtrless}$ in the following.

The quantities $\Omega_{\nu}^{\gtrless}$ are given by Eq. (55), however, to use this result we need to solve Eq. (49) for $r_{0}$. An alternative, and more convenient approach is to obtain an 
equation that determines $\Omega_{\nu}^{\gtrless}$ directly. By setting $m=0$ and $\mathcal{J}=\nu$ in Eq. (45) and using Eq. (44) to eliminate $r_{0}$ in favour of $\Omega$, we find that the desired equation is

$$
\left(\Omega^{2}-1-2 \gamma\right)\left[(\Omega-2 \nu)^{2}-1\right]+8 \gamma x_{B} \nu(\nu-\Omega)=0 .
$$

We now make a few key observations regarding the solutions to Eq. (63). First, for $\gamma$ and $x_{B}$ within the aforementioned range, Eq. (63) must yield two positive roots given by Eq. (55). In addition, the quartic in Eq. (63) is negative at $\Omega=0$, which along with the first observation guarantees that Eq. (63) has four real solutions, one negative and three positive. Finally, one can show that the two smallest positive roots are associated with the two negative $r_{0}$ roots of Eq. (49) and thus can be identified with $\Omega_{\nu}^{\gtrless}$.

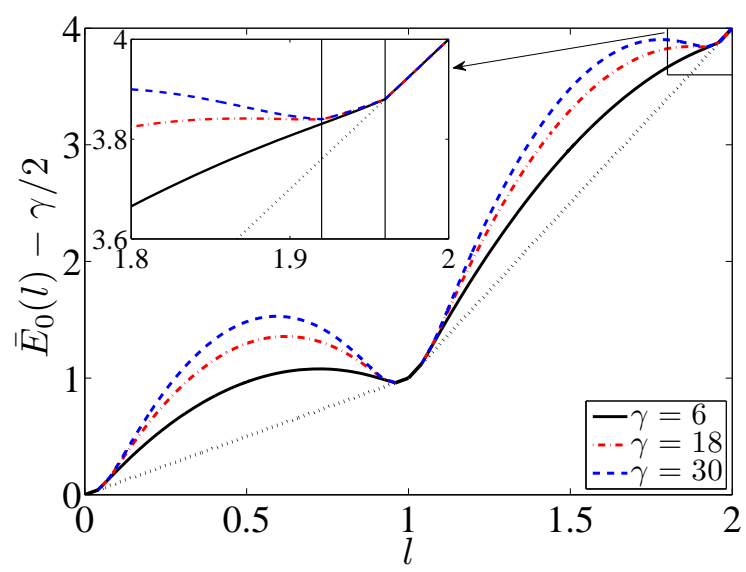

FIG. 7: Mean-field yrast spectrum for $\gamma=6,18$ and 30 at $x_{B}=0.04$. The inset is an expanded view of the behaviour of the energy curves in the vicinity of $l=2$. The linear lines from $l=0$ to 1 and from 1 to 2 correspond to the non-interacting yrast spectrum.

To see where $\Omega_{\nu}^{\gtrless}$ are located, we first consider the $x_{B}=$ 0 limit, in which case the solutions to Eq. (63) are $\Omega=$ $2 \nu \pm 1$ and $\Omega= \pm \sqrt{1+2 \gamma}$. Two of these four solutions correspond to $\Omega_{\nu}^{\gtrless}$ in the limit that $x_{B} \rightarrow 0$. Since $2 \nu-1$ is the smallest positive solution for $\gamma>2 \nu(\nu-1)$, we see that $\lim _{x_{B} \rightarrow 0} \Omega_{\nu}^{<}=2 \nu-1$. As for $\lim _{x_{B} \rightarrow 0} \Omega_{\nu}^{>}$, it takes the value $\min \{2 \nu+1, \sqrt{1+2 \gamma}\}$. Thus, if $2 \nu(\nu-1)<$ $\gamma \leq 2 \nu(\nu+1), \lim _{x_{B} \rightarrow 0} \Omega_{\nu}^{>}=\sqrt{1+2 \gamma}$. This implies that $\lim _{x_{B} \rightarrow 0} \Omega_{\nu}^{>}>2 \nu$ if $\gamma>\left(4 \nu^{2}-1\right) / 2$, which is the critical interaction strength for $x_{B}=0$ given in Eq. (57). For $x_{B} \neq 0$, the critical interaction strength can be obtained simply by inserting $\Omega=2 \nu$ into Eq. (63) and solving for $\gamma$. This leads to the result given in Eq. (58). For $\gamma$ slightly larger than $\gamma_{\mathrm{cr}, \nu}$, we have $\Omega^{<}\left(\nu-\nu x_{B}\right)<0$ and, since $\Omega_{\nu}^{<}<\Omega_{\nu}^{>}, \Omega^{>}\left(\nu-\nu x_{B}\right)>0$. In other words, we have established the possibility of a local minimum at $l=\nu x_{B}$.



FIG. 8: The angular velocity $\Omega(l)$ plotted as a function of $l$ for $l<2-2 x_{B}$.

Finally, we demonstrate that $\Omega^{<}\left(\nu-k x_{B}\right)>0$ for $k<\nu$ and for any $\gamma$ larger than $2 \nu(\nu-1)$. Using the argument given above, we have $\lim _{x_{B} \rightarrow 0} \Omega_{k}^{>}=\min \{2 k+$ $1, \sqrt{1+2 \gamma}$. If $\gamma>2 \nu(\nu-1), \lim _{x_{B} \rightarrow 0} \Omega_{k}^{>}=2 k+1$. Furthermore, it is not difficult to see from Eq. (63) that $\Omega_{k}^{>}<\lim _{x_{B} \rightarrow 0} \Omega_{k}^{>}=2 k+1$. Inserting this result into Eq. (62), we see that $\Omega^{<}\left(\nu-k x_{B}\right)>2 \nu-(2 k+1)>0$ if $k<\nu$. This proves that a local minimum cannot occur at $l=\nu-k x_{B}$ for $k<\nu$ for any value of $\gamma$. This result was established earlier for the case $\nu=2$ and $k=1[10,11]$.

To close this section, we emphasize that although a local minimum at $l=\nu x_{A}$ cannot occur if $\gamma<\gamma_{c r, \nu}$, it does not necessarily preclude the possibility of a local minimum at locations away from $l=\nu x_{A}$. To demonstrate this, we consider $x_{B}=0.04$ as an example. According to Eq. (59), local minima are possible at $l=\nu-\nu x_{B}$ for $\nu=1$ and 2. Let us focus on $\nu=2$. From Eq. (58) we see that the critical $\gamma$ value below which no local minimum appears at $l=2-2 x_{B}$ is $\gamma_{c r, 2}=125 / 6 \simeq 20.8$. In Fig. 7 we have obtained the mean-field yrast spectrum for a series of $\gamma$ values below and above this critical value. The qualitative behaviour proposed in our earlier work [11] is completely consistent with the quantitative results found here. The corresponding slope of the spectrum for $l \leq 2-2 x_{B}$ is shown in Fig. 8. For $\gamma=30>\gamma_{c r, 2}$, it is clear that the slope of the spectrum approaching $l=2-2 x_{B}$ from the left side is negative and there is a local minimum at this angular momentum value. For $\gamma=18<\gamma_{c r, 2}$, however, the slope at $l=2-2 x_{B}$ is positive, implying that the yrast spectrum does not have a local minimum at this angular momentum for this $\gamma$ value. But as we can see from Fig. 8, the slope decreases as $l$ moves away from $l=2-2 x_{B}$ and eventually passes through zero, indicating that a local minimum of the yrast spectrum occurs at some angular momentum $l<2-2 x_{B}$. 


\section{CONCLUSIONS}

In this paper, we have presented a comprehensive analysis of the mean-field yrast spectrum of a two-component gas in the ring geometry, using the analytic soliton solutions found in Refs. [7 9]. We find that the yrast spectrum reveals a complicated sequence of soliton states as a function of the interaction strength $\gamma$ and the population imbalance, specified by the minority concentration $x_{B}$. A denumerably infinite set of $x_{B}^{\text {crit. }}(\gamma, \mathcal{J})$ curves divides the $\gamma-x_{B}$ plane into regions, within each of which the yrast spectrum has a distinct structure. Our findings also demonstrate that the mean-field yrast spectrum of the two-component gas reduces to that of the singlecomponent system in a highly non-trivial way as $x_{B}$ tends to zero. Finally, the analysis of the yrast spectrum allows us to provide a definitive answer to the question of whether persistent currents at higher angular momenta can occur. In particular, we have derived an analytic formula for the critical interaction strength, above which persistent currents are found to exist at $l=\nu\left(1-x_{B}\right)$ for arbitrary integer $\nu$. It would clearly be of interest to see if these theoretical predications can be verified experimentally.

\section{Appendix A: Soliton solutions for the single-component gas}

Knowledge of the single-component soliton solutions (both attractive and repulsive) is necessary to understand the two-component case. In this appendix, we provide some useful information on these solutions, particularly on the evaluation of the quantities $\mathcal{M}_{s}$ and $W_{s}$ in Eqs. (43)-(44). Much of the material provided here can also be found in Refs. [5, 12, 13, 15].

The densities and phases of the single component solitons are given by Eqs. (38) and (43), which contain an unspecified elliptic parameter $m$. We will see that $m$ cannot assume all the values from 0 to 1 for either attractive or repulsive interactions. The allowable range of $m$ is determined by the requirement that the quantity $W_{s}^{2}$ is non-negative. For clarity we discuss seperately the case of attractive and repulsive interactions.

\section{Attractive interaction}

For $\gamma_{s}<0$, we see from its definition that $f_{s}<0$ since $K E-K^{2} \leq 0$. In view of Eq. (42) and the fact that $h_{s} \leq g_{s}$, we must have $h_{s} \leq 0$ and $g_{s} \geq 0$ in order that $W_{s}^{2} \geq 0$. This leads to the inequality

$$
K E-(1-m) K^{2} \leq \frac{\pi^{2}}{2 j^{2}}\left|\gamma_{s}\right| \leq K E
$$

Since the bounds of this inequality are monotonically increasing functions of $m$ (see Fig. 9), one finds

$$
m_{\min } \leq m \leq m_{\max },
$$

where $m_{\max }$ is the solution of equation $h_{s}=0$, while $m_{\text {min }}=0$ if $\left|\gamma_{s}\right|<2 j^{2} K(0) E(0) / \pi^{2}=j^{2} / 2$, and is otherwise the solution of the equation $g_{s}=0$.

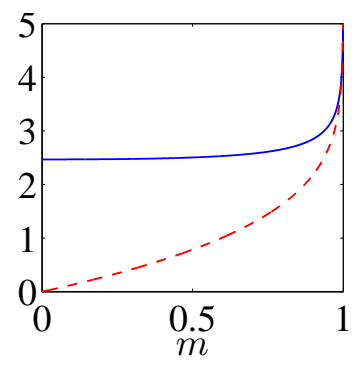

FIG. 9: $K E$ (solid line) and $K E-(1-m) K^{2}$ (dashed line) as a function of $m$.

To evaluate the quantity $\mathcal{M}_{s}$ for the allowed values of $m$ given by (A2), we need the complete elliptic integral of the third kind $\Pi\left(n_{s} ; K \mid m\right) \equiv \Pi\left(n_{s} \backslash \alpha\right)$, where $\alpha=$ $\sin ^{-1} \sqrt{m}$. For $\gamma_{s}<0$, one finds $m<n_{s}<1$ and the elliptic integral can be evaluated as [16]

$$
\Pi\left(n_{s} \backslash \alpha\right)=K+\frac{\pi}{2} \delta_{2}\left[1-\Lambda_{0}\left(\epsilon_{s} \backslash \alpha\right)\right],
$$

where $\delta_{2}=j K \sqrt{2 f_{s} /\left(g_{s} h_{s}\right)}, \epsilon_{s}=\sin ^{-1} \sqrt{h_{s} /\left[f_{s}(1-m)\right]}$ and $\Lambda_{0}$ is Heuman's Lambda function, defined as

$$
\Lambda_{0}\left(\epsilon_{s} \backslash \alpha\right)=\frac{2}{\pi}\left\{K E\left(\epsilon_{s} \backslash \pi / 2-\alpha\right)-[K-E] F\left(\epsilon_{s} \backslash \pi / 2-\alpha\right)\right\} .
$$

Here $F(\phi \backslash \alpha)$ and $E(\phi \backslash \alpha)$ are incomplete elliptic integrals of the first and second kind. Using Eq. (A3), one finds [5]

$$
\mathcal{M}_{s}(m)=\frac{1}{2 \pi}\left\{\sqrt{2 g_{s} h_{s} / f_{s}}+j \pi\left[1-\Lambda_{0}\left(\epsilon_{s} \backslash \alpha\right)\right]\right\} .
$$

To be specific, we now consider the case of $j=1$, namely a single soliton. In this case $\mathcal{M}_{s}(m)$ is a monotonically increasing function of $m$. At $m=m_{\max }$, we have $h_{s}=0$ which leads to $\epsilon_{s}=0$ and $\Lambda_{0}\left(\epsilon_{s} \backslash \alpha\right)=0$. Thus we find from Eq. (A5 that $\mathcal{M}_{s}\left(m_{\max }\right)=1 / 2$. Using Eq. (42) we also find that $W_{s}\left(m_{\max }\right)=0$. The lower bound of $\mathcal{M}_{s}(m)$ depends on the value of $\gamma_{s}$. For $\left|\gamma_{s}\right| \leq$ $1 / 2, m_{\min }=0$. At this value we have $g_{s}=\pi^{2} \gamma_{s}+\pi^{2} / 2$ and $f_{s}=h_{s}=\pi^{2} \gamma_{s}$. Thus we find $\epsilon_{s}=\pi / 2$ and $\Lambda_{0}=1$. Equation A5 then gives $\mathcal{M}_{s}(0)=\sqrt{2 \gamma_{s}+1} / 2$. From Eq. (42) we find that $\left|W_{s}(0)\right|=\sqrt{2 \gamma_{s}+1} / 2 \pi$. Now, for $\left|\gamma_{s}\right|>1 / 2, m_{\min }$ is the solution to $g_{s}=0$. At this value we have $\sqrt{h_{s} /\left[f_{s}(1-m)\right]}=1$ and thus $\epsilon_{s}=\pi / 2$. Using the Legendre's relation

$$
E F\left(\frac{\pi}{2} \backslash \frac{\pi}{2}-\alpha\right)+E\left(\frac{\pi}{2} \backslash \frac{\pi}{2}-\alpha\right) K-K F\left(\frac{\pi}{2} \backslash \frac{\pi}{2}-\alpha\right)=\pi / 2
$$


we find $\Lambda_{0}(\epsilon \backslash \alpha)=1$. Thus we obtain $\mathcal{M}_{s}\left(m_{\min }\right)=0$ and $W_{s}\left(m_{\text {min }}\right)=0$ for $\left|\gamma_{s}\right|>1 / 2$. As a concrete example, we show $\mathcal{M}_{s}$ calculated for $\gamma_{s}=-0.3$ and $\gamma_{s}=-0.7$ in Fig. 10.
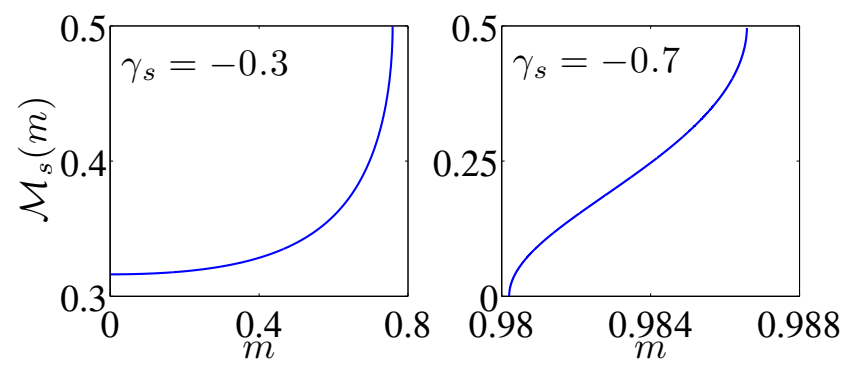

FIG. 10: $\mathcal{M}_{s}(m)(j=1)$ as a function of $m$ for $\gamma_{s}=-0.3$ and $\gamma_{s}=-0.7$.

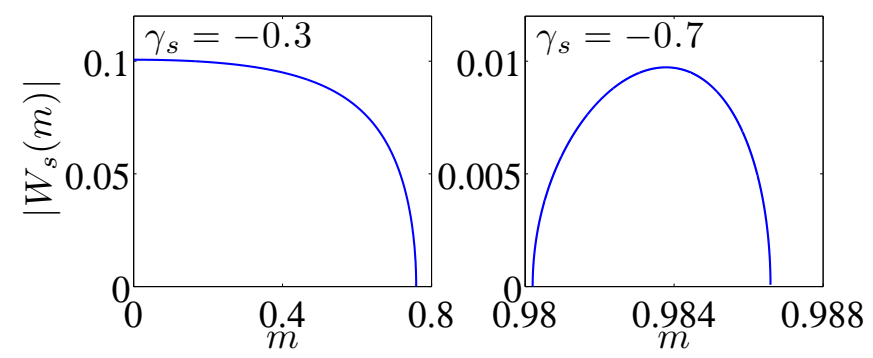

FIG. 11: $\left|W_{s}(m)\right|(j=1)$ as a function of $m$ for $\gamma_{s}=-0.3$ and $\gamma_{s}=-0.7$.

Once $\mathcal{M}_{s}$ and $W_{s}$ have been determined, various physical quantities such as energy, angular momentum and angular velocity can be calculated using the formulae given in Sec. II. For example, according to Eq. (44) and the fact that $0 \leq \mathcal{M}_{s} \leq 1 / 2$ for $\gamma_{s}<0$, the range of angular velocity values is given by $2 J_{s}-1 \leq \Omega \leq 2 J_{s}+1$. This implies the following important relation for the attractive gas [15]

$$
J_{s}=\left[\frac{\Omega}{2}+\frac{1}{2}\right]
$$

where $[x]$ denotes the largest integer less than or equal to $x$.

\section{Repulsive interactions}

For $\gamma_{s}>0$, and using the fact that $K E-(1-m) K^{2} \geq$ 0 , we find from their definitions that $g_{s} \geq h_{s}>0$. Because $h_{s} \geq f_{s}$, we must impose the condition $f_{s} \geq 0$ in order for $W_{s}^{2} \geq 0$, namely $\pi^{2} \gamma_{s}+2 j^{2} K E-2 j^{2} K^{2} \geq 0$. In view of the fact that $K^{2}-K E$ is a monotonically increasing function of $m$, we obtain the allowable range of

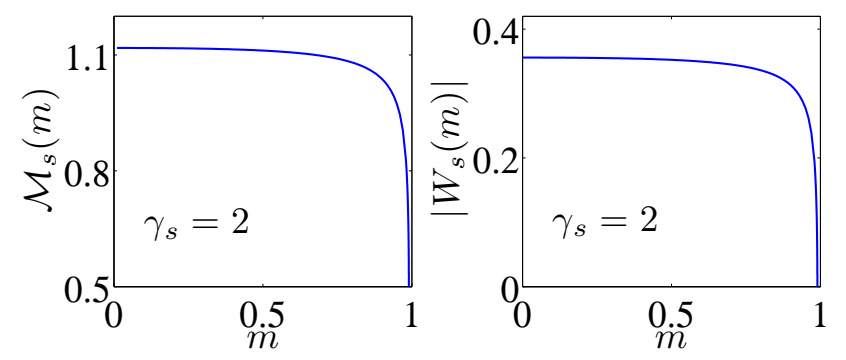

FIG. 12: $\mathcal{M}_{s}(m)$ (left) and $W_{s}(m)$ (right) as a function $m$ for $j=1$ and $\gamma_{s}=2$.

$m$ as $0 \leq m \leq m_{\max }$, where $m_{\max }$ is the solution to the equation $f_{s}=0$.

Now, in the case of $\gamma_{s}>0$, we have $n_{s}<0$. The evaluation of $\Pi\left(n_{s} \backslash \alpha\right)$ for $n_{s}<0$ can be reduced to the case of $m<n_{s}^{\prime}<1$ [16], where $n_{s}^{\prime} \equiv\left(m-n_{s}\right)\left(1-n_{s}\right)^{-1}=$ $m g_{s} / h_{s}$. One finds

$$
\Pi\left(n_{s} \backslash \alpha\right)=K \frac{f_{s}}{g_{s}}+2 j^{2}(1-m) K^{2} \frac{f_{s}}{g_{s} h_{s}} \Pi\left(n_{s}^{\prime} \backslash \alpha\right),
$$

where $\Pi\left(n_{s}^{\prime} \backslash \alpha\right)$ can be evaluated as before and is given by

$$
\Pi\left(n_{s}^{\prime} \backslash \alpha\right)=K+\frac{\pi}{2} \delta_{2}^{\prime}\left[1-\Lambda_{0}\left(\epsilon_{s}^{\prime} \backslash \alpha\right)\right]
$$

Here $\delta_{2}^{\prime}=\sqrt{g_{s} h_{s} / 2 f_{s}} /[j(1-m) K]$ and $\epsilon^{\prime}=$ $\sin ^{-1} \sqrt{f_{s} / h_{s}}$. Using Eqs. (A8) and (A9) we obtain for $\gamma_{s}>0$

$$
\mathcal{M}_{s}(m)=\frac{1}{2 \pi}\left\{\sqrt{2 g_{s} f_{s} / h_{s}}+j \pi\left[1-\Lambda_{0}\left(\epsilon_{s}^{\prime} \backslash \alpha\right)\right]\right\} .
$$

The quantity $\mathcal{M}_{s}(m)$ for the repulsive gas is a monotonically decreasing function of $m$. For $j=1$ and at $m=0$, we have $f_{s}=h_{s}=\pi^{2} \gamma_{s}$ and $g_{s}=\pi^{2} \gamma_{s}+\pi^{2} / 2$. Using these values we find $\epsilon_{s}^{\prime}=\pi / 2$ and $\Lambda_{0}\left(\epsilon_{s}^{\prime} \backslash \alpha\right)=1$. This leads to $\mathcal{M}_{s}(0)=\frac{1}{2} \sqrt{2 \gamma_{s}+1}$. At $m=m_{\max }$, we find $f_{s}=0, \epsilon_{s}^{\prime}=0$ and $\Lambda_{0}\left(\epsilon_{s}^{\prime} \backslash \alpha\right)=0$. Thus we obtain $\mathcal{M}_{s}\left(m_{\max }\right)=1 / 2$. Similarly we find $0 \leq\left|W_{s}(m)\right| \leq$ $\sqrt{2 \gamma_{s}+1} / 2 \pi$. An illustration of the behaviour of $\mathcal{M}_{s}(m)$ and $\left|W_{s}(m)\right|$ for $\gamma_{s}=2$ is shown in Fig. 12.

As in the case of attractive interaction, various physical quantities of interest can be obtained after $\mathcal{M}_{s}(m)$ and $\left|W_{s}(m)\right|$ have been determined. Here we only point out an oversight made in Ref. [5] regarding the range of the angular velocity $\Omega$. We first observe that although the phase winding number $J_{s}$ in Eq. (44) can take any integer value, one can without loss of generality restrict its value to $J_{s}=0,1$, which can be shown to correspond to the solutions with angular momenta in the range $0 \leq l \leq 1$. Taking $J_{s}=0$ and $\operatorname{sgn} W_{\mathrm{s}}=-1\left(\operatorname{sgn} W_{\mathrm{s}}=1\right.$ renders $\Omega$ and $l_{s}$ negative ), we find from Eq. (44) that $1 \leq \Omega=$ $2 \mathcal{M}_{s}(m) \leq \sqrt{2 \gamma_{s}+1}$. Since $-\sqrt{2 \gamma_{s}+1} / 2 \pi \leq W_{s} \leq 0$, we find $0 \leq l_{s}=\pi W_{s}+\Omega / 2 \leq 1 / 2$ for $J_{s}=0$, where 
$l_{s}=0$ at $m=0$ and $l_{s}=1 / 2$ at $m=m_{\max }$. A similar analysis of $J_{s}=1$ yields $2-\sqrt{2 \gamma_{s}+1} \leq \Omega \leq 1$ for $1 / 2<$ $l_{s} \leq 1$. We thus obtain $2-\sqrt{2 \gamma_{s}+1} \leq \Omega \leq \sqrt{2 \gamma_{s}+1}$ for $0 \leq l_{s} \leq 1$. This shows that the range of $\Omega$ corresponding to an integral interval of $l_{s}$ in fact depends on $\gamma_{s}$ and in general cannot be restricted to $0 \leq \Omega \leq 2$ as claimed in Ref. [5].

Lastly, we provide a useful discussion of the soliton wave functions at $l_{s}=0$ and $l_{s}=1 / 2$. For the former we have $m=0, \Omega=\sqrt{2 \gamma_{s}+1}$ and $J_{s}=0$. Since $\operatorname{dn}(u \mid m=0)=1$, we find from Eq. (38) that $\rho_{s}(\theta)=1 / 2 \pi$. Furthermore, at $m=0$ one finds $\Pi\left(n_{s} ; K\left(\theta-\theta_{0}\right) / \pi, m\right)=\left(\theta-\theta_{0}\right) / 2$. Using this result in Eq. (43) we obtain $\varphi_{s}(\theta)-\varphi_{s}\left(\theta_{0}\right)=l_{s}\left(\theta-\theta_{0}\right)=0$. Thus the wave function is simply $\psi_{s}(\theta)=1 / \sqrt{2 \pi}$. This is the expected plane wave solution at $l_{s}=0$. For $l_{s}=1 / 2$, we have $m=m_{\max }, \Omega=1$ and $J_{s}=0$. Since in this case $f_{s}=0$ and $g_{s}=2 K^{2}$, we find from Eq. (39) that $\eta_{s}=-1$. The density is thus given by

$$
\rho_{s}(\theta)=\frac{K^{2}}{\pi^{3} \gamma_{s}}\left[1-\operatorname{dn}^{2}\left(\frac{j K}{\pi}\left(\theta-\theta_{0}\right) \mid m\right)\right]
$$

Since $\operatorname{dn}(u=0 \mid m)=1$, we see that at $\rho_{s}\left(\theta_{0}\right)=0$ which implies that this is a dark soliton.

\section{Acknowledgment}

This work was supported by a grant from the Natural Sciences and Engineering Research Council of Canada.
[1] F. Bloch, Rhys. Rev. A 7, 2187 (1973).

[2] The yrast terminology was introduced into cold-atom physics in B. Mottelson, Phys. Rev. Lett. 832695 (1999).

[3] E. Lieb, Phys. Rev. 130, 1616 (1963).

[4] M. Ishikawa and H. Takayama, J. Phys. Soc. Jpn. 49, 1242 (1980).

[5] R. Kanamoto, L. D. Carr, and M. Ueda, Phys. Rev. A 79, 063616 (2009).

[6] R. Kanamoto, L. D. Carr, and M. Ueda, Phys. Rev. A 81, 023625 (2010).

[7] A. V. Porubov and D. F. Parker, Wave Motion 29, 97 (1999).

[8] J. Smyrnakis, M. Magiropoulos, A. D. Jackson, and G. M. Kavoulakis, J. Phys. B 45, 235302 (2012).

[9] J. Smyrnakis, M. Magiropoulos, G. M. Kavoulakis and A. D. Jackson, Phys. Rev. A 87, 013603 (2013).

[10] J. Smyrnakis, S. Bargi, G. M. Kavoulakis, M. Magiropou- los, K. Karkkainen, and S. M. Reimann, Phys. Rev. Lett. 103, 100404 (2009).

[11] K. Anoshkin, Z. Wu, and E. Zaremba, Phys. Rev. A 88, 013609 (2013).

[12] L. D. Carr, Charles W. Clark, and W. P. Reinhardt, Phys. Rev. A 62, 063610 (2000).

[13] L. D. Carr, Charles W. Clark, and W. P. Reinhardt, Phys. Rev. A 62, 063611 (2000).

[14] R. S. Irving, Integers, Polynomials, and Rings: A Course in Algebra (Springer, 2004).

[15] R. Kanamoto, H. Saito, and M. Ueda, Phys. Rev. A 68, 043619 (2003).

[16] M. Abramowitz and I. A. Stegun, Handbook of Mathematical Functions (National Bureau of Standards, Washington, DC, 1964). 